\title{
Competencias informacionales en los estudios de grado en enfermería: el caso español
}

\author{
César Manso-Perea*, Aurora Cuevas-Cerveró**, Sergio González-Cervantes*** \\ * Biblioteca. Colegio oficial de Enfermería de Madrid (CODEM) \\ Correo-e: biblioteca@codem.es | ORCID iD: https://orcid.org/0000-0001-5266-7868 \\ ** Facultad Ciencias de la Documentación (UCM \\ Correo-e: macuevas@ucm.es | ORCID iD: https://orcid.org/0000-0002-0928-0403 \\ *** Escuela Universitaria Enfermería Cruz Roja Madrid (EUE CREM-UAM) \\ Correo-e: sergio.gonzalez@ucavila.es | ORCID iD: https://orcid.org/0000-0002-9058-6126
}

Recibido: 26-03-2018; 2a versión: 24-07-2018; Aceptado: 29-08-2018

Cómo citar este artículo/Citation: Manso-Perea, C.; Cuevas-Cerveró, A.; González-Cervantes, S. (2019). Competencias informacionales en los estudios de grado en enfermería: el caso español. Revista Española de Documentación Científica, 42 (1): e229. https://doi.org/10.3989/redc.2019.1.1578

Resumen: Este trabajo pretende analizar la inclusión de contenidos relacionados con competencias informacionales en los planes de estudio de Grado en enfermería en España. La elección de este escenario nos permitirá conocer la importancia que los diferentes centros otorgan a estas competencias en sus planes de estudio y obtener una visión sobre cómo se enfoca la integración de esos contenidos a partir de las guías docentes de las asignaturas. Se realiza una investigación de tipo exploratorio y descriptivo. La muestra final incluye 118 centros de 58 universidades y 325 asignaturas, pertenecientes al curso 2014-2015. Se observan las diferencias que cada centro otorga a esta inclusión. Son casi inexistentes los itinerarios curriculares completos con este tipo de contenidos. Se considera necesario incidir en la importancia de diseñar programas de ALFIN para enfermería específicos en cada centro, en los que estos contenidos queden recogidos de forma progresiva en diferentes asignaturas por curso.

Palabras clave: Competencias informacionales; alfabetización informacional; estudios de grado; enfermería; bibliotecas universitarias.

\section{Informational competencies in Nursing Degree: the Spanish case}

Abstract: The aim of this research is to analyze the inclusion of contents related to informational competences in the Nursing Degree's curricula in Spain. The choice of this scenario will allow us to know the importance that the different centers grant to these competences in their study plans and obtain a vision on how the integration of these contents starting from the teaching guides of the subjects. It is an exploratory and descriptive research. The final sample contains 118 centers of 58 universities and 325 subjects, belonging to the 2014-2015 academic year. The differences that each center grants to this inclusion are observed. The complete curricular itineraries with this type of contents are almost non-existent. It is considered necessary to influence the importance of designing specific nursing Information Literacy programs in each center, in which contents are progressively included in different subjects per academic year.

Keywords: Information competences; information literacy; degree studies; nursing; university libraries.

Copyright: @ 2019 CSIC. Este es un artículo de acceso abierto distribuido bajo los términos de la licencia de uso y distribución Creative Commons Reconocimiento 4.0 Internacional (CC BY 4.0). 


\section{INTRODUCCIÓN}

Tratar de alfabetización en la actualidad implica aderezar el término con un gran abanico de matices: digital, informacional, audiovisual y multimedia entre otros, convirtiéndolo en metáfora de una amplia gama de competencias que no parecen tener fin.

Las competencias que los estados y las instituciones educativas deben promover han sido enunciadas por las principales instituciones políticas, educativas culturales y bibliotecarias, como la Comisión Europea, UNESCO e IFLA y todas ellas coinciden en la necesidad de fomentar habilidades para acceder, seleccionar, usar, evaluar y comunicar la información para poder transformarla en conocimiento y adaptarnos a un entorno altamente mediatizado por la tecnología y la sobreabundancia de información. Este conjunto de habilidades se ha denominado desde los años 70 del pasado siglo information literacy, en español alfabetización en información o informacional (ALFIN).

Las declaraciones de la Sorbona (1998), Bolonia (1999), Praga (2001), Berlín (2003), Bergen (2005) entre otras, promovieron una revisión en profundidad de los Sistemas de Educación Superior en la Unión Europea dando lugar a una reforma estructural sin precedentes conocida como Espacio Europeo de Educación Superior (EEES). El modelo educativo que promueve el EEES está centrado en el aprendizaje por competencias de tal forma que todos los aspectos curriculares y organizativos se han de definir en torno a las competencias que debe adquirir el alumno (Biggs, 2005).

A pesar de las exigencias del EEES de un modelo competencial está siendo difícil para el profesorado la adaptación y cambio de prácticas docentes que se habían mantenido milenariamente. Este modelo "competencial" ha desplazado a una enseñanza tradicionalmente centrada en la transmisión de conocimientos y ha supuesto un cambio paradigmático en la práctica docente y discente.

Al llegar a la universidad, los estudiantes requieren una formación básica para interactuar con el proceso informacional, puesto que el desarrollo de sus habilidades no es suficiente para poder localizar, acceder, recuperar, evaluar y usar de forma crítica la información de forma autónoma. Estas competencias son denominadas por los teóricos de diferentes formas: competencias informacionales, digitales y multimedia, entre otras, y son el objeto de la alfabetización informacional.

En España hay dos organismos que sobresalen en la promoción de la alfabetización informacional desde el ámbito bibliotecario, la Red de Bibliote- cas Universitarias (REBIUN), comisión sectorial de la Conferencia de Rectores de las Universidades Españolas (CRUE) en la que están representadas todas las bibliotecas universitarias y científicas españolas y el Consejo de Cooperación Bibliotecaria (CCB), órgano dependiente del Ministerio de Cultura que canaliza la cooperación bibliotecaria entre las administraciones públicas.

REBIUN ha apoyado la necesidad de potenciar las competencias en gestión de la información y su desarrollo transversal en el modelo docente promovido por el Espacio Europeo de Educación Superior (EEES) en el cual el alumno se convierte en protagonista de su propio aprendizaje sustentado en la adquisición de competencias. Desde 2007 hasta la actualidad REBIUN ha transitado por diferentes paradigmas conceptuales, desde la alfabetización informacional, pasando por las competencias informáticas e informacionales (CI2) y Media and Information Literacy (MIL) hasta llegar a su actual adscripción al modelo DigComp que aboga por la denominación competencia digital.

En el II Plan Estratégico 2007-2010 de REBIUN se incluye la ALFIN en la línea estratégica 1 Competencias en Información (Programa ALFIN) cuyo objetivo era potenciar y promover acciones para el desarrollo e implementación de las competencias en información como competencias transversales en el nuevo modelo docente. En abril de 2009, mientras las universidades estaban embarcadas en los nuevos planes de estudios del EEES, la Comisión mixta CRUE-TIC y REBIUN (2013) publican el informe Competencias informáticas e informacionales en los estudios de grado, planteando la necesidad de integrar las competencias informáticas e informacionales en las nuevas titulaciones de nuestro país, pues la ordenación de las enseñanzas universitarias oficiales, descrita en el RD 1393/2007 y en el RD $861 / 2010$, introducía el concepto de competencia quedando recogida en las recomendaciones de los libros blancos de los títulos de grado de la ANECA. Es en este momento cuando en REBIUN acuñan el acrónimo $\mathrm{CI} 2$, con el interés de potenciar la idea de que ambas competencias, informáticas e informacionales, están íntimamente relacionadas entre sí y son fundamentales para desenvolverse satisfactoriamente en la sociedad de la información.

En 2014 REBIUN hace público un trabajo exhaustivo de definición de indicadores recogido en el documento titulado "Definición de Competencias Informaciones (CI)", en el que organiza las Competencias Informacionales en 5 competencias, estableciendo para cada una de ellas las realizaciones competenciales y los indicadores, conocimientos, procedimientos y actitudes necesarios para alcanzar dicha competencia. Actualmente REBIUN se ha 
decantado por el modelo promovido por la Unión Europea denominado Digcomp. En 2010 la Comisión Europea le encarga al Instituto de Prospectiva Tecnológica (IPTS) de la Comisión Europea el desarrollo del marco DigComp, el marco de competencias digitales para la ciudadanía. El procedimiento para la realización de DigComp fue complejo y sometido a validación, se comenzó con una fase de una revisión de los estudios existentes y recopilación de datos, análisis de casos y un cuestionario a 95 expertos-, y continuó con una fase de consulta y validación del marco por parte de stakeholders a partir de debates online, aportaciones de expertos, jornadas, seminarios y conferencias. Con las aportaciones recibidas en este proceso, IPTS publicó en 2013 el marco completo contrastado, con orientaciones de aplicación. El informe final salió a mitad del 2013 firmado por Anusca Ferrari. Del 2015 al 2016 se contrastó la primera versión y se publicó la versión 2.0 con algunas modificaciones. En 2017 se ha publicado la versión 2.1. DigComp define la competencia digital como el conjunto de conocimientos, habilidades, actitudes, estrategias y sensibilización que se requieren cuando se utilizan las TIC y los medios digitales con el objetivo de realizar tareas, resolver problemas, comunicarse, gestionar información, colaborar, crear y compartir contenidos, y construir conocimiento de manera efectiva, eficiente, adecuada, crítica, creativa, autónoma, flexible, ética y reflexiva para el trabajo, el ocio, la participación, el aprendizaje, la socialización, el consumo y el apoderamiento (Ferrari, 2013). Este modelo integra las competencias ALFIN, la alfabetización mediática y las competencias informáticas, además de otras competencias generales o relativas al aprendizaje con medios colaborativos, participativos y sociales. Se trata de una iniciativa cuyo objetivo es identificar y describir los componentes claves de la competencia digital (DC) en términos de conocimiento, habilidades y actitudes, procurando aunar la multiplicidad de iniciativas dispersas y diversas en un marco común que aúne competencias informacionales, digitales, mediáticas y sociales, ofreciendo una herramienta para la implementación, medición, desarrollo del currículo, competencias del profesorado, certificación y autoevaluación. Con el valor añadido de ser un marco que no permanece estático sino que va evolucionando e incluyendo mejoras según se va testando y la sociedad va evolucionando.

Digcomp plantea un nuevo paradigma para REBIUN a la hora de entender la formación, e introduce nuevos conceptos: competencia digital, alfabetización digital, e-habilidades y alfabetización mediática. La adscripción de REBIUN al nuevo modelo les ha llevado a revisar el anterior basado en las Competencias Informáticas e Informacionales.
Dado su interés en adaptarse al nuevo modelo REBIUN ha transformado el documento Definición de Competencias Informaciones (CI) en otro denominado Equivalencia de descriptores de la Competencia Digital (DIGCOMP) con el Decálogo $\mathrm{CI} 2^{1}$ en el que estudian ambos modelos estableciendo las equivalencias y armonizando los documentos.

A pesar de la importancia de las competencias informacionales y digitales en la educación universitaria y la gran implicación y esfuerzo de REBIUN, la integración estratégica y planificada no se ha producido y actualmente los proyectos para integrar la ALFIN en el currículo académico son desiguales, sobre todo porque este tipo de iniciativas se han realizado de manera no formal, ya sea por la actitud proactiva y el entusiasmo de los bibliotecarios que buscan la colaboración en los docentes o también al contrario, los docentes que buscan la colaboración de los bibliotecarios.

Ante este escenario, diferentes trabajos han planteado posibles estrategias para integrar la ALFIN en el sistema educativo (Area, 2007; Basili, 2008; Comisión Mixta CRUE-TIC, REBIUN, 2009; Gomes y Hernández-Perez, 2013; Pirela y otros, 2014; CCB, 2018; en ellos se especifican las formas de abordar la ALFIN en la educación y todos destacan la necesidad y urgencia de afrontar este reto.

Al especificar las formas de abordar la ALFIN en la educación superior se utiliza diferente terminología que es necesario aclarar para entender mejor la situación en la que transcurre. Se emplea el término extracurricular como un curso fuera del currículo académico, es decir, no relacionado con ninguna materia. Intercurricular es utilizado para sesiones, seminarios, cursos o conferencias programadas dentro del currículo académico de una titulación, Intracurricular para una ALFIN integrada a una titulación mediante la colaboración entre profesores y bibliotecarios, y por último se utiliza el término stand-alone para cursos independientes dentro del plan de estudio (Wang, 2011)

Una de las propuestas más comunes para la enseñanza de la ALFIN en la universidad es ofertar asignaturas optativas en los planes de estudio de introducción a la documentación especializada. En España comenzaron a introducirse en las titulaciones de ciencias de la salud a principios de los años noventa (Gómez Hernández y Benito, 2001). En 2011, un estudio realizado por la Universidad de Zaragoza recogía que en seis grados: Ciencias Ambientales, Medicina, Odontología, Nutrición y Dietética, Filosofía y Periodismo aparecía una asignatura específica de documentación científica que abordaba competencias informacionales (Salvador y otros, 2011). 
En contra de estos últimos modelos de integración de la ALFIN en la universidad, algunos autores señalan que para que tenga más relevancia, la formación de la ALFIN debe ser obligatoria y no voluntaria u optativa. La formación en competencias informacionales "debe ser una formación obligatoria para todo el alumnado y no responder a solicitudes concretas y aisladas de determinados profesores más afines a la biblioteca, lo que lleva a una formación desigual para el alumnado y no garantiza que todos reciban esta formación" (González Fernández-Villavicencio, 2010). Utilizar los trabajos académicos, como los Trabajos Fin de Grado sería otra oportunidad para integrar las competencias informacionales en el plan de estudios en la universidad En concordancia con esta idea los trabajos de Limberg, y otros (2008) y Bent y Stockdale (2009) señalan que si la formación de ALFIN se imparte a estudiantes que tienen un trabajo de investigación designado, los estudiantes encuentran un aliciente, el nivel de participación aumenta y en consecuencia, obtienen mejores resultados. Algunos autores avalan la idea de integrar la ALFIN de forma gradual, a través de los cursos durante todo el Grado (Badke, 2008) y que, por lo tanto, cuánto más tiempo se dedica en los estudios de Grado, mayor es el aprendizaje y mejores son los resultados (Detlor y otros, 2010).

Desde la perspectiva de los estudiantes, Pinto y Puertas (2012) en un estudio demostraron que cuanto más nivel creen que tienen adquirido en una competencia, mayor importancia le dan los estudiantes a esa competencia. Las mismas autoras argumentan que "[...] probablemente cuanto más se entrene al alumnado en una competencia determinada y mayor nivel vayan adquiriendo en ella, mayor será la importancia y el valor que le den a la misma".

Entendemos con González Fernández-Villavicencio (2010) que uno de los factores a tener en cuenta en las diferentes opciones de integrar la ALFIN en el currículo académico a través de asignaturas radica en desarrollar el itinerario de la ALFIN de manera ordenada, secuencial y progresiva basándose en las necesidades de información de los estudiantes en sus años de Grado y evolucionando hasta el postgrado. Otra cuestión relevante planteada por González Fernández-Villavicencio (2016) es la necesidad de establecer alianzas entre los implicados, profesores, bibliotecarios y otras instancias educativas como departamentos y decanatos. Es evidente que este planteamiento debe contar con el beneplácito de las políticas institucionales, que reconozcan la ALFIN como una de las prioridades de aprendizaje institucional, valorando el trabajo interdisciplinar. Con apoyo institucional, con la colaboración de to- dos los actores de la comunidad universitaria y sobre todo con actitud bibliotecaria, la integración de las competencias informacionales en la formación del estudiante universitario resultará más factible.

Los documentos emitidos por instituciones nacionales e internacionales que analizan prospectivas y tendencias en la educación superior y en las bibliotecas han destacado la relevancia de promover las competencias informacionales.

El informe Prospectiva 2020 reúne la visión del Grupo de Trabajo Estratégico para el Estudio de prospectiva y sobre la biblioteca en un entorno informacional y social del CCB de España y define las 10 circunstancias que más van a afectar a los diversos tipos de bibliotecas hasta 2020; en el apartado número ocho destaca que la educación, el aprendizaje y las habilidades serán la clave de la misión de las Bibliotecas y establece como uno de los retos a alcanzar el normalizar la alfabetización informacional como un servicio más de la biblioteca, dotándola de visibilidad y recursos (CCB, 2018).

Cada dos años, el Comité de Planificación y Análisis de Investigación de ACRL publica un documento sobre las tendencias principales en la educación superior y su relación con las bibliotecas universitarias. ACRL justificaba en su propuesta del año 2000 la integración curricular de la ALFIN en la educación superior al exponer que "el logro de las aptitudes para el acceso y uso de la información exige comprender que esta gama de habilidades no es algo extraño al plan de estudio, sino que está entretejida en el contenido, estructura y secuencia del curriculum" (ACRL/ALA, 2000). Otros autores como Bundy (2004), Rockman y otros (2004); Lau y Cortés (2006) y Area (2007) respaldaban este planteamiento. En el documento de 2018 Tendencias en bibliotecas universitarias las principales tendencias señaladas por ACRL priorizan nuevas propuestas de alfabetización en el marco para la Alfabetización en Información, la gestión de datos de investigación (RDM), edición académica digital, las tendencias de evaluación de la colección, fusiones de proveedores de contenidos o evidencias de aprendizaje (ACRL, 2018).

El Proyecto Horizon del New Media Consortium (NMC) investiga y publica desde hace más de una década Informes que especifican las tendencias de la tecnología emergente en el ámbito educativo. Se acaba de publicar la tercera edición del informe que NMC dedica a las bibliotecas académicas y de investigación de todo el mundo el NMC HORIZON REPORT 2017 LIBRARY (Becker y otros, 2017). ¿Qué encontrarán las bibliotecas académicas y de investigación en los próximos cinco años?, ¿Qué tendencias y tecnologías impulsarán el cambio?, 
¿Cuáles son los retos fundamentales y cómo podemos plantear soluciones? Estas preguntas sobre la adopción de tecnología y el cambio educativo condujeron los debates de setenta y siete expertos que elaboraron este informe, traducido al español por la UOC. Entre los principales retos que denominan Retos asumibles (comprensibles y resolubles) destacan la accesibilidad a servicios y recursos de las bibliotecas y competencias profesionales que lo hagan posible y la mejora de la alfabetización digital especificando que las bibliotecas tienen la oportunidad de liderar la alfabetización digital.

\section{Antecedentes de la ALFIN en la formación de enfermería}

La literatura anglosajona sobre la integración de competencias informacionales en el plan de estudios de enfermería es abundante. La inclusión de un programa de ALFIN en los estudios de enfermería en la universidad de Wollongong (Australia) fue evaluada y presentada en un artículo donde se evidenciaba la eficacia del mismo (Wallace y otros, 1999). Con menos éxito, en un principio, la Escuela de enfermería de la Universidad Estatal de San Francisco desarrolló, implementó y evaluó un programa integrado de ALFIN en su plan de estudios. La integración incluyó una variedad de estrategias de instrucción programadas en los diferentes semestres. Sin embargo, los estudiantes no percibieron que tuvieran más éxito en el acceso a la información (Verhey, 1999). Bibliotecarios y docentes de la Universidad Ball State, en Indiana diseñaron un método para la ALFIN en la especialidad de enfermería. El programa requería a los estudiantes una serie de competencias establecidas previamente y estaba orientado a que los estudiantes obtuvieran habilidades informativas para sus trabajos de investigación y en su desarrollo profesional (Dorner y otros, 2001). La División de Enfermería de la Universidad de Nueva York emprendió una iniciativa curricular para integrar componentes de la ALFIN en todos los cursos, con el objetivo de promover la práctica basada en la evidencia. En colaboración con la biblioteca, diseñaron módulos de instrucción en habilidades informacionales pertinentes para cada curso. Los contenidos estaban enfocados a la búsqueda bibliográfica, a la metodología de la investigación y al manejo de bases de datos y otros recursos en ciencias de la salud (Jacobs y otros, 2003). Otro ejemplo desarrollado en la Escuela de Tecnología de Queensland en Australia, integraba los componentes informacionales en el plan de estudios con aumentos graduales en las complejidades de las actividades de aprendizaje (Barnard y otros, 2005). Las habilidades de escritura académica se incluyeron en un diseño curricular de ALFIN integrado en el plan de estudios de enfer- mería de la Universidad de Hong Kong, junto con las búsquedas bibliográficas, la cita y referencia de documentos y el manejo de bases de datos y otros recursos en ciencias de la salud (Tarrant y otros, 2008). La búsqueda bibliográfica, el manejo de bases de datos y contenidos relacionados con la metodología de investigación en enfermería orientados a la práctica basada en la evidencia son los contenidos más propuestos en las diferentes estrategias de integración de ALFIN en los planes de estudio por lo que se deprende de diferentes iniciativas (Jacobsen y Andenaes, 2011; Bønløkke y otros, 2012; Janke y otros, 2012 y Stombaugh y otros, 2013; Özbıçakçı y otros, 2015; Argüelles, 2016).

Por lo que respecta a España cabe destacar el análisis de la competencia informacional en las ciencias de la salud en el marco de las universidades españolas (Ortoll, 2004). El estudio pone de manifiesto el grado de presencia de asignaturas relacionadas con dicha competencia, y el enfoque y tratamiento de las mismas. Para ello se analizan los planes de estudios de las carreras relacionadas con ese ámbito de enseñanza, y entre ellas enfermería.

En cuanto a la integración curricular de competencias informacionales en los planes de estudio de enfermería, la Universidad Rovira i Virgili incluyó una asignatura de 4,5 créditos en el curso 20032004. La asignatura Fundamentos de búsqueda y selección de documentación electrónica estaba orientada al acceso y evaluación de la información académica en internet. Antes de esta asignatura, "la única formación sobre gestión de la información, se llevaba a cabo desde la biblioteca. Eran sesiones de formación de usuarios, dirigidas a orientar sobre el uso de los recursos bibliotecarios. Por otro lado, no había habido ninguna reivindicación por parte de los alumnos sobre la insuficiencia de formación que se les estaba proporcionando para buscar y seleccionar información". (García, 2010). La biblioteca de la Escuela Universitaria de Enfermería de Cruz Roja en Madrid (EUE CREM-UAM) diseñó una propuesta de itinerario curricular para la formación en competencias informacionales. Esta propuesta se implementó con la participación del personal de la misma en la docencia. Se estructuró en 4 niveles de complejidad (Manso y otros, 2015). Cada nivel se integra en una asignatura por curso (Bioestadística y Tecnologías de la Información y de la Comunicación [TIC], Bases y metodología en enfermería comunitaria, Metodología de la investigación y Trabajo Fin de Grado). Los contenidos de dicha formación versaban sobre búsqueda bibliográfica, bases de datos y otros recursos en ciencias de la salud, cita y referencia, trabajo académico, etc. Actualmente la propuesta se está implementando en la institución mencionada y próximamente se presentarán los resultados. 
Por su parte las bibliotecas de ciencias de la salud de la Universidad de Alcalá han implementado la competencia gestión de la información en los estudios de enfermería desde el año 2009 hasta la actualidad en la asignatura Búsqueda y gestión de información en ciencias sociosanitarias y en la asignatura Trabajo fin de Grado (Dominguez Aroca, 2017).

\section{COMPETENCIAS INFORMACIONALES EN CIENCIAS DE LA SALUD}

En relación con las enseñanzas en ciencias de la salud en general y enfermería en particular, se incluye la competencia transversal Gestión de la información en los libros blancos de la disciplina.

Existen varios propulsores para su desarrollo en los planes de estudio. El reconocimiento de las habilidades relacionadas con el manejo de la información para desenvolverse con éxito en la Sociedad de la Información y el Conocimiento, como un aprendizaje a lo largo de la vida, no sólo como ya se ha mencionado, en relación al reconocimiento de las habilidades, sino también ligado a la propia formación como se ha visto, y por otro lado, y más ligado a la propia formación del estudiante, el desarrollo del pensamiento crítico, la solución de problemas y la aparición de la práctica de la medicina y enfermería basadas en la evidencia.

Las actividades de los profesionales de enfermería, ya sean en la etapa universitaria o posteriormente en la práctica profesional en el ámbito de la asistencia clínica, de la gestión, de la investigación o de la educación, son intensivas en información, estando presentes muchos elementos informacionales (Ortoll, 2004). Los estudiantes se enfrentan a "nuevos retos pedagógicos, más orientados a la práctica, basados en la propia iniciativa, y en la adquisición de destrezas para las búsquedas, el tratamiento y la organización de la información científica disponible" (Guerra y otros, 2014). Es imprescindible el papel activo de los estudiantes en todas las actividades formativas tanto en el aula como en las prácticas clínicas. Para ello, necesitan contar con una serie de competencias y habilidades que les permitan localizar y gestionar la información de manera eficaz. En este escenario los estudiantes, además, necesitan habilidades en el uso de las TIC para fortalecer su trabajo autónomo que queda consolidado en la utilización de entornos virtuales de aprendizaje. Esto requiere de individuos cualificados en las competencias de uso y dominio de la información. "El profesional sanitario, tal como sucede en otros entornos, comienza su ejercicio profesional tras un periodo reglado de formación, y trabaja en un sistema que le provee de medios materiales para el desarrollo de su actividad. Sin embargo existe una diferencia notable con otras profesiones en relación a sus necesidades de información, que podemos centrar en dos cuestiones: La repercusión que sus decisiones tienen sobre la salud de las personas y el acelerado ritmo de innovación científica en todas las facetas relacionadas con la salud" (García y otros, 2011). Otro aspecto que ilustra la importancia del acceso y uso de la información en los profesionales enfermeros es la mejora de la calidad de los cuidados en base a una práctica basada en la evidencia. En nuestro caso la Enfermería Basada en la Evidencia (EBE), es un entorno que reafirma la importancia de las competencias informacionales como garantes para la búsqueda y acceso a las mejores evidencias científicas disponibles para apoyar la toma de decisiones.

Además, deben conocer las herramientas que se encuentran a su disposición, para que puedan ser competentes en la gestión de la producción científica sobre su disciplina. Uno de los aspectos que se ha visto modificado, una vez constatada la irrupción de las TIC y el desarrollo de la sociedad de la información y el conocimiento, es la relación que se establece entre el profesional de enfermería y el paciente. Los pacientes tienen cada vez más posibilidades de acceder a la información en ciencias de la salud. Pueden consultar tratamientos alternativos, nuevas técnicas para su cuidado, información sobre fármacos, etc. Esto requiere de un profesional solvente en el acceso a la información y en el conocimiento de recursos que respondan a esas necesidades de información del paciente.

En consecuencia, la formación universitaria debe proporcionar a los estudiantes y futuros profesionales de la disciplina enfermera, el desarrollo de habilidades y estrategias para saber cuándo y por qué se necesita información, dónde encontrarla, cómo evaluarla, cómo utilizarla y cómo comunicarla de manera ética.

En este escenario, diversas universidades y organismos han recogido normas relacionadas con las competencias informacionales en ciencias de la salud. Nos encontramos con un documento que sugiere una lista de competencias específicas para los estudiantes de enfermería elaboradas a partir de las normas de la ACRL. Estas competencias se clasifican en habilidades elementales, habilidades específicas de la disciplina, habilidades relacionadas con el uso de recursos electrónicos y habilidades de investigación avanzadas (Humboldt State University Library, 1999). Otra iniciativa fue el Proyecto Nightingale de la Comunidad Europea, que expone un amplio programa de formación en competencia informática e informacional. 
En 2011, el Royal College of Nursing (RCN) publicó un documento sobre las competencias ALFIN en salud: Finding, using, and managing information: nursing, midwifery, health and social care information literacy competences. Basado en los principios de la ALFIN de Australia y Nueva Zelanda, establecía siete competencias (RCN, 2011). Por su parte, la ACRL presentó Information Literacy Competency Standards for Nursing (ACRL/ALA, 2013), basados en las aptitudes para el acceso y uso de la información en la educación superior publicadas en el año 2000. Las normas se refieren a las habilidades de información que necesitan los estudiantes de enfermería en su vida académica y su posterior desarrollo profesional.

En el contexto latinoamericano es evidente el alcance obtenido en materia de competencias informacionales en salud como se desprende de diferentes propuestas (Fernández Valdés y Zayas, 2016; González Valiente y otros, 2013; Cisneros, 2010; Sánchez Diaz, 2010).

\section{OBJETIVOS}

Una vez justificada la importancia de integrar las competencias informacionales en el currículo académico en el Grado en enfermería, este artículo pretende analizar el nivel de inclusión de contenidos relacionados con competencias informacionales en los planes de estudio de Grado en enfermería en España. La elección de este escenario nos permitirá conocer una amplia perspectiva sobre la importancia que los diferentes centros otorgan a las competencias informacionales en sus itinerarios curriculares. Asimismo, podremos obtener una visión sobre cómo se enfoca la integración de esos contenidos a través de una serie de categorías.

\section{METODOLOGÍA, MATERIALES EMPLEADOS Y FUENTES}

Según hemos podido identificar en la literatura científica, nuestro trabajo indaga en un tema no investigado anteriormente por lo que podemos identificarlo como exploratoria. El método descriptivo es usado en ciencia para describir las características de un fenómeno, sujeto o población a estudiar. En la medida en que perseguimos identificar y especificar características, propiedades, y rasgos que nos permitan identificar en qué estado se encuentra la incorporación de las competencias informacionales en los estudios de Grado en Enfermería podemos definir nuestra investigación como descriptiva.

El análisis de contenidos se sustentó desde la perspectiva de Krippendorff (citado por Andreu, 2002), que lo define como "una técnica de investi- gación destinada a formular, a partir de ciertos datos, inferencias reproducibles y válidas que puedan aplicarse a su contexto".

Para la realización de este trabajo se han revisado los contenidos de los planes de estudio de Grado en enfermería de las universidades españolas a través de la información que aparece en las guías docentes que recogen los sitios web de los centros donde se imparte la titulación. Incidimos especialmente en tres asignaturas que integran contenidos en competencias informacionales ( $y$ que a partir del marco regulador de la titulación de Grado en enfermería pueden ser susceptibles de alojar contenidos relacionados con competencias informacionales): Bioestadística y TIC, Metodología de la investigación y Trabajo Fin de Grado (TFG), en sus diferentes denominaciones. De igual modo, también se revisan iniciativas en forma de asignaturas, que incluyen contenidos en materia de competencias informacionales en los diferentes centros en los que se imparte.

El universo de investigación consta de 123 centros. La muestra final incluye 118 centros y 325 asignaturas, perteneciente a los planes de estudio de grado en enfermería implantados en España en el curso 2014-2015, representando un 95,93\% la tasa de resultado sobre el total pues de este análisis se han excluido los centros que no tenían sus guías docentes accesibles en sus sitios Web.

Para tratar la información obtenida, dado el volumen de información, se construyó una base de datos ad hoc, llamada CIENF (Figura 1) formada por los siguientes campos: Nombre de asignatura, URL, Tipo de asignatura, Curso, Horas de dedicación a contenidos ALFIN, Integración en otra asignatura, Presencia ALFIN, Formación del docente, Contenidos, Centro, Universidad, URL y Comunidad Autónoma, Observaciones, Lugares de formación, Métodos docentes y Competencias específicas de la asignatura.

Una vez identificadas las asignaturas con presencia de contenidos ALFIN, analizamos el enfoque de esos contenidos. Las guías docentes incluyen para cada asignatura la descripción de los contenidos que se van a impartir. A través de ese análisis se observó que se utilizaba una gran variedad de denominaciones para contenidos idénticos. En consecuencia, se determinó normalizar esos contenidos, estableciendo unas categorías que sintetizaran todos los contenidos de las diferentes asignaturas. Para designar las categorías se utilizaron, además del contenido de las propias guías docentes de las asignaturas, diferentes modelos orientados a salud; el modelo IDEIAS (Cuevas y García, 2010), los del Royal College of Nursing, sobre las compe- 
Figura 1. Registro de la Base de Datos CIENF

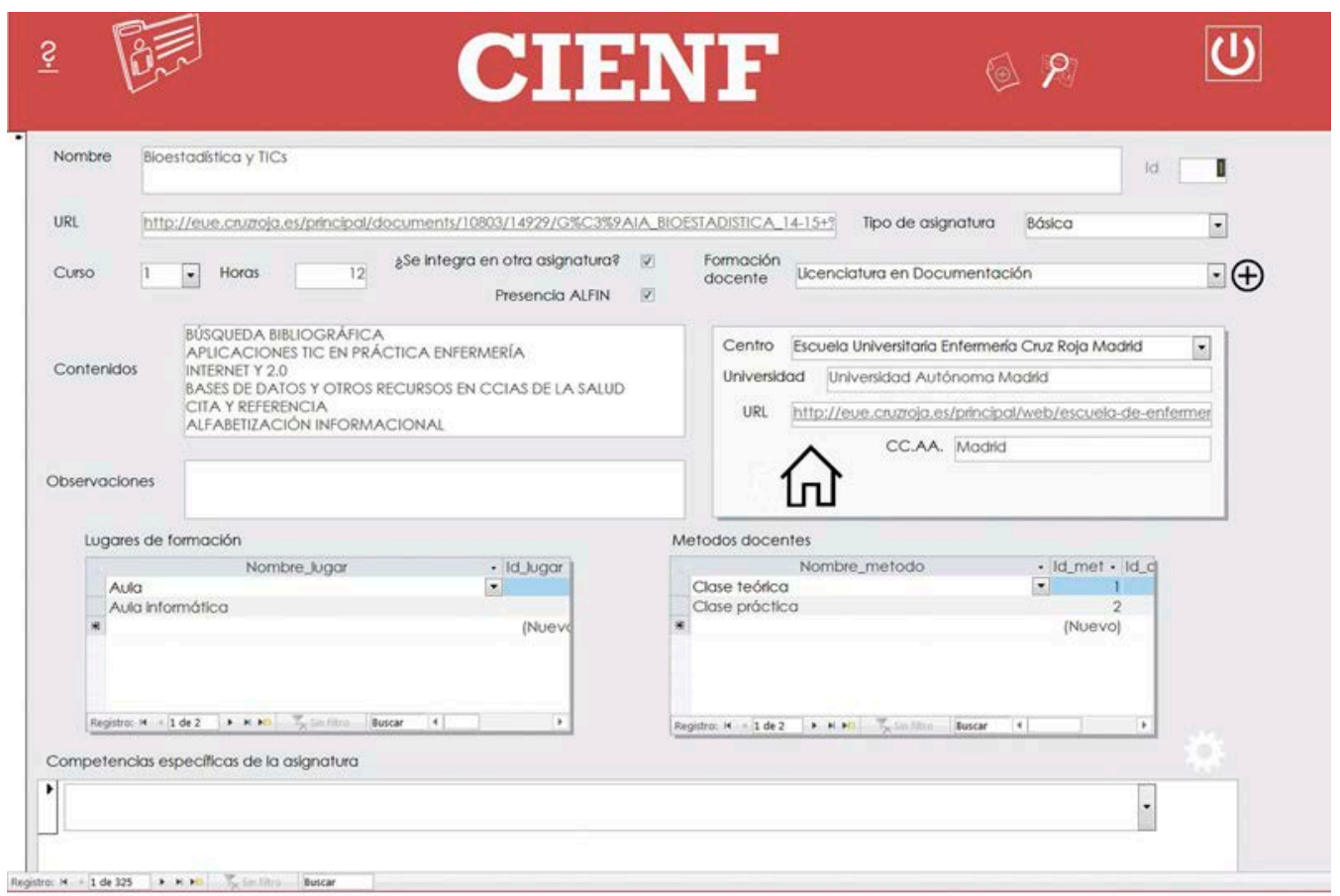

tencias Alfin: Finding, using, and managing information: nursing, midwifery, health and social care information literacy competences y las normas ALFIN para enfermería de la ACRL. Finalmente se establecieron 14 categorías: Búsqueda bibliográfica, Aplicaciones TIC en la práctica de enfermería, Internet y 2.0, Informática, Metodología de la investigación, Bases de datos y otros recursos en ciencias de la salud, Entornos virtuales de enseñanza aprendizaje, Ética de la información, Cita y referencia, Alfabetización informacional, Documentación, Uso de la biblioteca, Trabajo académico y Difusión de la investigación en enfermería.

A partir del análisis de asignaturas con presencia ALFIN y del enfoque que le dan a sus contenidos relacionados con las competencias informacionales en enfermería, vimos necesario analizar otras variables. Es preciso saber el tipo de asignatura (básica, obligatoria, optativa) y curso para saber el tratamiento que recibe dentro del itinerario curricular. Otros aspectos que se analizaron fueron el tipo de presencia. Es decir, si se integran los contenidos en otra asignatura o son asignaturas completas, lo que nos indicará la exhaustividad con la que se impartirán esos contenidos. También se analizaron las comunidades autónomas y universidades con presencia ALFIN para representar un mapa de ALFIN en enfermería que nos daría una visión de las tendencias que éstas proponen para incorporar la competencia Gestión de la información en los planes de estudio de Grado en enfermería.

El tratamiento de los datos y su análisis estadístico se realizó con el Software IBM $®$ SPSS $®$ Statistics V21.0.0., realizándose un análisis descriptivo de las categorías estudiadas.

\section{RESULTADOS Y DISCUSIÓN}

En base a los criterios especificados en el apartado de metodología, el número total de asignaturas analizadas fue de 325, distribuidas éstas en 58 universidades. En lo que a distribución nacional se refiere, el mayor número de asignaturas analizadas se localiza en Cataluña con 50 asignaturas (ver Tabla I).

En lo que a distribución por cursos se refiere, el mayor número de asignaturas analizadas se localiza en el primer curso con 144, seguido muy de cerca de cuarto con 133 asignaturas analizadas.

Por último, atendiendo al tipo de asignatura, para el total de la muestra, 134 eran básicas, 154 obligatorias y 37 optativas.

De las 325 asignaturas analizadas, en 186 están presentes, en mayor o menor medida, contenidos relacionados con la ALFIN. Por su parte, en lo que respecta a si se integran los contenidos en otra asignatura o son asignaturas completas, que nos 
Tabla I. Asignaturas analizadas por Comunidad Autónoma

\begin{tabular}{|l|c|c|}
\hline & Asignaturas analizadas & Porcentaje \\
\hline Andalucía & 39 & 3,7 \\
\hline Aragón & 12 & 1,8 \\
\hline Asturias & 6 & 2,8 \\
\hline Baleares & 9 & 3,1 \\
\hline Canarias & 10 & 0,9 \\
\hline Cantabria & 3 & 11,4 \\
\hline Castilla y León & 37 & 4,6 \\
\hline Castilla-La Mancha & 15 & 15,4 \\
\hline Cataluña & 50 & 0,6 \\
\hline Ceuta & 2 & 4,9 \\
\hline Extremadura & 16 & 6,2 \\
\hline Galicia & 20 & 0,6 \\
\hline La Rioja & 2 & 11,4 \\
\hline Madrid & 37 & 0,9 \\
\hline Melilla & 3 & 3,7 \\
\hline Murcia & 12 & 3,4 \\
\hline Navarra & 11 & 2,5 \\
\hline País Vasco & 8 & 10,2 \\
\hline Valencia & 33 & \\
\hline Total & 325 & \\
\hline
\end{tabular}

indicarán la exhaustividad con la que se impartirán esos contenidos y la dedicación, observamos que la ALFIN se desarrolla sobre todo en asignaturas más amplias, es decir, sus contenidos se integran en otra asignatura. Esta integración de contenidos como parte de una asignatura se presenta en 114 asignaturas (un $61,3 \%$ ) de las asignaturas con presencia ALFIN.

Si consideramos la tipología de las asignaturas donde se imparten contenidos con presencia de ALFIN, observamos que la inmensa mayoría de asignaturas serán de tipo básica $(43,5 \%)$ y obligatoria $(40,3 \%)$, mientras que las de menor presencia serán las de tipo optativa $(16,1 \%)$.

Por su parte, si tenemos en cuenta el curso donde se imparten contenidos con presencia de ALFIN, advertimos que es en $1^{\circ}(50 \%)$ donde habitan más asignaturas con este tipo de contenidos. Si bien la asignaturas son básicas, el estudiante al iniciar sus estudios empieza a tener necesidades de información. Es en $4^{\circ}(32,3 \%)$ curso con la asignatura TFG donde la presencia de estos contenidos se muestra en segundo lugar. Podemos considerar que es en el TFG donde se pone de manifiesto que se han alcanzado las competencias vinculadas al título, y entre ellas la competencia Gestión de la información.

Ahondando en la presencia ALFIN para cada una de las Comunidades, podemos observar que la Comunidad que mayor presencia ALFIN presenta es Canarias, para la cual, el $100 \%$ de las asignaturas estudiadas tiene presencia ALFIN. Por el contrario, Castilla-La Mancha es la Comunidad que menor presencia ALFIN presenta respecto a las asignaturas estudiadas $(26,7 \%)$. (Ver tabla II).

Una vez analizados las asignaturas que incluyen contenidos relacionados con la ALFIN podemos observar que, de entre las categorías establecidas para describir esos contenidos, es Bases de datos y otros recursos en ciencias de la salud la categoría que más se destina para ese fin con un $(18,3 \%)$, seguido de Búsqueda bibliográfica con un (17,8\%). Por su parte la que menor presencia tiene es Alfabetización Informacional con un 1,7\% de presencia. (Ver Figura 2). 
Tabla II. Frecuencia ALFIN y porcentaje sobre el total de asignaturas

\begin{tabular}{|l|c|c|c|}
\hline & $\begin{array}{c}\text { Asignaturas } \\
\text { analizadas }\end{array}$ & Frecuencia ALFIN & $\begin{array}{c}\text { Porcentaje ALFIN } \\
\text { sobre el total }\end{array}$ \\
\hline Andalucía & 39 & 17 & 43,6 \\
\hline Aragón & 12 & 5 & 41,7 \\
\hline Asturias & 6 & 3 & 50,0 \\
\hline Baleares & 9 & 3 & 33,3 \\
\hline Canarias & 10 & 10 & 100,0 \\
\hline Cantabria & 3 & 2 & 66,7 \\
\hline Castilla y León & 37 & 21 & 56,8 \\
\hline Castilla-La Mancha & 15 & 4 & 26,7 \\
\hline Cataluña & 50 & 32 & 64,0 \\
\hline Ceuta & 2 & 1 & 50,0 \\
\hline Extremadura & 16 & 13 & 68,8 \\
\hline Galicia & 20 & 1 & 65,0 \\
\hline La Rioja & 2 & 23 & 50,0 \\
\hline Madrid & 37 & 1 & 62,2 \\
\hline Melilla & 3 & 4 & 33,3 \\
\hline Murcia & 12 & 8 & 33,3 \\
\hline Navarra & 11 & 6 & 72,7 \\
\hline País Vasco & 33 & & 75,0 \\
\hline Valencia & & 21 & 63,6 \\
\hline
\end{tabular}

Figura 2. Porcentaje de la presencia de contenidos relacionados con la ALFIN

Bases de datos y otros recursos en ciencias de la salud

Búsqueda bibliográfica

- Búsqueda bibliográfica

Cita y referencia

Informática

Internet y 2.0

Aplicaciones TIC en práctica enfermera

Documentación

Difusión de investigación en enfermeria

Ética de la información

- Trabajo académico

Uso biblioteca

Entornos virtuales de enseñanza aprendizaje

alfabetización informacional

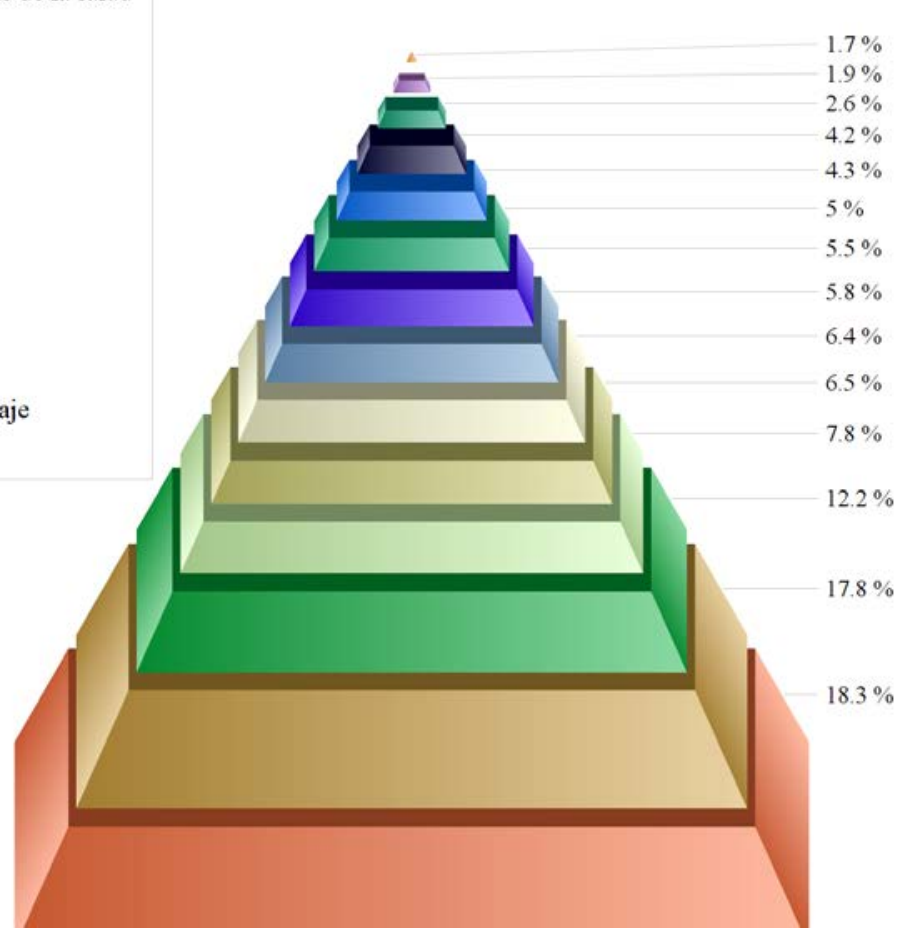


Las categorías de análisis que se proponen pretenden describir los contenidos que integren competencias informacionales y que ayuden a explicar ese enfoque de inclusión en la estructura de la asignatura, ya sea completa o integrada en otra asignatura. Por consiguiente, el enfoque de las asignaturas se ha estructurado de acuerdo con el análisis de 14 categorías.

La categoría Bases de datos y otros recursos en ciencias de la salud es la que más aparece para integrar la competencia informacional. Esta categoría trata aspectos vinculados con recursos bibliográficos que tienen relación con las ciencias de la salud y enfermería. Bases de datos como PubMed, CINAHL, Cuiden, etc. $Y$ otros recursos para buscar información relacionada con la disciplina enfermera.

Por su parte la segunda categoría sería Búsqueda bibliográfica con un $17,8 \%$. Esta categoría abordaría los contenidos referentes a la planificación de una búsqueda bibliográfica.

Un $12,2 \%$ de las asignaturas analizadas se destinan a la categoría Metodología de la investigación. Esta categoría tiene como objetivo que el estudiante identifique la labor investigadora con la disciplina enfermera para iniciarse en la investigación científica. Abarcaría contenidos de práctica basada en la evidencia, planteamiento y tipos de investigación, lectura crítica, etc.

Con un $7,8 \%$ Cita y referencia es la cuarta categoría más utilizada para la formación de contenidos relacionados con las competencias informacionales en el Grado en enfermería. Contenidos como los estilos de cita y referencias bibliográficas, Vancouver o APA o el uso de Gestores de referencias bibliográficas, por ejemplo.

La categoría Internet y 2.0 comprende un 6,5\% de las asignaturas. Los contenidos de esta categoría versan sobre Internet y navegadores, redes y aplicaciones 2.0 útiles para la disciplina enfermera.

Al igual que la categoría anterior, Informática abarca un $6,5 \%$. Los temas que sugiere esta categoría son todos aquéllos relacionados con herramientas ofimáticas como procesadores de texto, hojas de cálculo, etc.

Un $5,8 \%$ de las asignaturas analizadas forman parte de la categoría Aplicaciones TIC en práctica enfermera. Esta categoría englobaría los contenidos necesarios para aplicar las Tecnologías de la Información y la Comunicación (TIC) en los diferentes ámbitos de la profesión enfermera como los procesos asistenciales, la e-salud, Teleasistencia, APPs, aplicaciones dirigidas a pacientes $\mathrm{y} / \mathrm{o}$ profesionales, etc.
Por su parte, un 5,5\% tiene un enfoque documental que se correspondería con la categoría Documentación, que englobaría aspectos como el concepto y áreas de la documentación, tipos de documentos, análisis documental, etc. Las asignaturas diseñadas con esta categoría tienen como objetivo que los estudiantes se familiaricen con conceptos y terminología relacionada con la Documentación.

Difusión de investigación en enfermería, categoría que abarcaría todos los contenidos implicados en el proceso de publicación en la disciplina enfermera, obtuvo un $5 \%$ en el análisis.

Un 4,3\% se destina a Ética de la información o contenidos relacionados con los aspectos éticos en las publicaciones y trabajos de los estudiantes, derechos de autor, plagio, buen uso de la información o derechos de los ciudadanos en materia de información y documentación clínica.

La elaboración de trabajos académicos, redacción escrita y expresión oral son algunos contenidos que recoge la categoría Trabajo académico con sólo un 4,2\%.

Sólo un 2,6\% de las asignaturas están enfocadas al Uso de la biblioteca ya a contenidos como el manejo del catálogo.

Finalmente las categorías con menos presencia en el análisis sobre el enfoque de las asignaturas en función del contenido impartido en las mismas fueron, Entornos virtuales de enseñanza aprendizaje y Alfabetización informacional con un 1,9\% y $1,7 \%$ respectivamente. Esta última categoría que sería el eje vertebrador del estudio abarcaría contenidos acerca de su concepto.

Un análisis posterior de los planes de estudio desde el curso 2014-2015 a la actualidad, evidenció escasas modificaciones en los contenidos de las guías docentes. No obstante empiezan a destacar contenidos relacionados con la identidad digital, el posicionamiento y la promoción de la actividad investigadora de los profesionales de enfermería en asignaturas como Metodología de la investigación y TFG. Estas modificaciones no solo se refieren a los contenidos sino también al aumento en el número de horas para su impartición. En consecuencia se establece un aumento relativo al enfoque de la categoría Metodología de la investigación con respecto al análisis inicial.

\section{CONCLUSIONES}

A la luz de los resultados, se asume el papel clave de la competencia Gestión de la información en la formación y el ejercicio profesional enfermero, lo que otorga una especial relevancia a la integración 
de contenidos relacionados con competencias informacionales en los estudios de Grado en enfermería.

En relación a las asignaturas susceptibles de alojar este tipo de contenidos, sólo un $57,3 \%$ de las 325 asignaturas analizadas tiene presencia de estos contenidos. Entre ellas, en un $61,3 \%$ la presencia de ALFIN es como parte de los contenidos, por lo que sólo un $38,7 \%$ se integran en el plan de estudios como asignaturas con contenidos informacionales de forma exclusiva.

Estas asignaturas dedicadas exclusivamente a ALFIN tienen en un 16,1\% carácter optativo, lo que no garantiza el aprendizaje de estos contenidos por parte de todos los estudiantes. Para evitar esto, puede ser recomendable la modalidad integrada.

En referencia al curso en el que estos contenidos están presentes, destacan las asignaturas de $1^{\circ}$ y 40 de grado. Esto se relaciona con que los estudiantes toman conciencia de sus necesidades de información al iniciar sus estudios y no las retoman hasta $4^{\circ}$, en la asignatura de TFG.

Es por ésto que se considera necesario distribuir transversalmente este tipo de contenidos en las distintas asignaturas por curso y niveles de complejidad. La competencia Gestión de la información no puede vincularse a un curso determinado, sino que su desarrollo y adquisición debe ser progresiva, en un proceso de evolución en distintos niveles de complejidad.

Si atendemos al enfoque con el que son tratadas las asignaturas a partir de sus contenidos, destacan categorías como Búsqueda bibliográfica, Bases de datos y otros recursos en ciencias de la salud, Metodología de la investigación y Cita y referencia. Categorías estas más ligadas a la formación de usuarios tradicional, o a la práctica basada en la evidencia, como en el caso de metodología de la investigación.

Destaca el escaso porcentaje obtenido por categorías como Ética de la información o Alfabetización informacional, siendo esta última, la que menos presencia ha obtenido. Se considera de vital importancia sensibilizar al estudiante de enfermería sobre los conceptos de ALFIN y competencia informacional, ya que no se puede adquirir una competencia si no se le explica en qué consiste y con qué problemas de información puede encontrarse en su itinerario académico y profesional.

A raíz del análisis, es posible convenir las diferencias que cada centro otorga a la importancia en la inclusión de este tipo de contenidos. Si bien es cierto que son casi inexistentes los itinerarios curriculares completos con este tipo de contenidos, se considera necesario incidir en la importancia de diseñar programas de ALFIN para enfermería específicos en cada centro, en los que estos contenidos queden recogidos de forma progresiva en diferentes asignaturas por curso.

Por último se ha observado que el escenario actual de una acción de ALFIN para la formación de Grado en enfermería en España es: contenidos integrados en una asignatura básica de primer curso, relacionados con la búsqueda bibliográfica, bases de datos y otros recursos en ciencias de la salud y la metodología de investigación en la disciplina enfermera.

\section{RECOMENDACIONES}

Considerando que el estudiante necesita interactuar intensamente con la información durante el desarrollo de la titulación de Grado en enfermería, se recomienda el reconocimiento y defensa de la ALFIN en la titulación de Grado en enfermería como una competencia genérica necesaria que deben adquirir y afianzar los estudiantes. Esto requiere la inserción de la ALFIN en el itinerario curricular y el desarrollo de un plan estratégico, tanto de la universidad como de la biblioteca universitaria, para formalizar la inclusión de esta formación en los centros que imparten la disciplina enfermera.

Según se desprende de los resultados obtenidos en esta investigación, proponemos la implantación de la ALFIN de manera sistemática, en la titulación de Grado en enfermería incorporando las acciones formativas a los contextos de aprendizaje tanto del aula como de la clínica, de forma secuencial a lo largo de los cursos y transversal en el plan de estudios, para garantizar su alcance a todos los estudiantes.

Finalmente, sería conveniente concienciar a la comunidad universitaria de la importancia de incluir contenidos en las asignaturas del Grado en enfermería relacionados con el posicionamiento y la visibilidad de la labor investigadora en la disciplina.

\section{NOTAS}

1. Equivalencia de descriptores de la Competencia Digital (DIGCOMP)con el Decálogo CI2. Disponible en: http:// unesdoc.unesco.org/images/0021/002127/212715s. pdf [Consulta: 23-2-2018]. 


\section{REFERENCIAS}

ACRL/ALA. (2000). Information Literacy Competency Standards for Higher Education. Boletín de la Asociación Andaluza de Bibliotecarios, (60), 93-110. Traducción: Cristóbal Pasadas. Normas sobre aptitudes para el acceso y uso de la información en la educación superior.

ACRL/ALA. (2013). Information Literacy Competency Standards for Nursing. Recuperado de: http://www.ala. org/acrl/standards/nursing [Consulta 15/01/2019].

ACRL. (2018). 2018 top trends in academic libraries: a review of the trends and issues affecting academic libraries in higher education. Recuperado de: https://crln. acrl.org/index.php/crlnews/article/view/17001/18750 [Consulta 15/01/2019].

Andreu, J. (2002). Las técnicas de análisis de contenido: una revisión actualizada. Recuperado de: http://public.centrodeestudiosandaluces.es/pdfs/S200103.pdf [Consulta 15/01/2019].

Area Moreira, M. (2007). Documento marco de Rebiun para la CRUE: adquisición de competencias en información: una materia necesaria en la formación universitaria. La Laguna. Recuperado de: https://www. rebiun.org/sites/default/files/2017-11/Resumen_Documento marco_REBIUN_Adquisicion\%20competencias_Manuel_Area_2007.pdf [Consulta 15/01/2019].

Argüelles, C. (2016). Curriculum-integrated information literacy (CIIL) in a community college nursing program: a practical model. Community College Journal of Research and Practice, 40(11), 942-953. https:// doi.org/10.1080/10668926.2016.1147395

Badke, W. (2008). Ten reasons to teach information literacy for credit. Online, 32(6), 47-9. Recuperado de: http://home.ubalt.edu/ub78145/My\%20Library/ storage/XA8J94CW/badke_10\%20reasons\%20to\%20 teach \%20info\%20lit\%20for\%20credit_2008.pdf [Consulta 15/01/2019].

Basili, C. (2008). Information Literacy policies and developments in Higher Education institutions in Europe. En VI Jornadas CRAI: Los Centros de Recursos para el aprendizaje y la Investigación: Las competencias en información en las nuevas enseñanzas universitarias, Pamplona. Recuperado de: http://www.unav.edu/ documents/1807770/2776220/crai6.pdf. [Consulta $15 / 01 / 2019]$.

Barnard, A.; Nash, R.; O`Brien, M. (2005). Information literacy: developing lifelong skills through nursing education. Journal of Nursing Education, 44(11), 505-510.

Becker, S. A., Cummins, M., Davis, A., Freeman, A., Hall, C. G., \& Ananthanarayanan, V. (2017). NMC horizon report: 2017 higher education edition. The New Media Consortium.

Bent, M.; Stockdale, E. (2009). Integrating information literacy as a habit of learning -assessing the impact of a golden thread of IL in the curriculum. Journal of Information Literacy, 3(1), 43-50. https://doi. org/10.11645/3.1.212
Biggs, J.B. (2005). Calidad del aprendizaje universitario. Madrid: Narcea.

Bønløkke, M.; Kobow, E.; Kristensen, A. K. (2012). Curriculum Integrated Information Literacy-a Challenge. Nordic Journal of Nursing Research, 32(3), 53-55. https://doi.org/10.1177/010740831203200311

Bundy, A. (2004). Australian and New Zealand information literacy framework: principles, standards and practice. $2^{a}$ ed. Recuperado de: http://archive.caul. edu.au/info-literacy/InfoLiteracyFramework.pdf. [Consulta 15/01/2019].

Cisneros Velázquez, S. (2010). Evaluación de la alfabetización informacional en el sector de la salud. Anales de documentación. 13, 41-51.

Comisión mixta CRUE-TIC y REBIUN (2009). Las competencias informáticas e informacionales en los estudios de grado. Recuperado de: http://www.uv.es/websbd/ formacio/ci2.pdf. [Consulta 09/03/2016].

Comisión mixta CRUE-TIC y REBIUN (2013). Manual para la formación de competencias informáticas e informacionales. Traducción y adaptación de: Handbook for Information Literacy Teaching, Universidad de Cardiff. Recuperado de: https://www.rebiun.org/sites/default/files/2017-11/Manual_formacion_CI2_completo_2013.pdf [Consulta 15/01/2019].

CCB. Consejo de Cooperación bibliotecaria (2018). Informe Prospectiva 2020. Recuperado de: http://www. ccbiblio.es/wp-content/uploads/Estudio_prospectiva_2020.pdf [Consulta 15/01/2019].

Cuevas Cerveró, A.; García Moreno, M. A. (2010). Ideias, un modelo de evaluación para inclusión digital y alfabetización informacional orientado a salud. El profesional de la información. 19(3), 240-245. Recuperado de: http://www.elprofesionaldelainformacion.com/contenidos/2010/mayo/03.pdf [Consulta 15/01/2019].

Detlor, B. ; Julien, H.; Serenko, A.; Booker, L. (2010). Factors affecting student learning outcomes of information literacy instruction. Proceedings of the American Society for Information Science and Technology, 47(1), 1-2. Recuperado de: https://onlinelibrary.wiley.com/doi/pdf/10.1002/ meet.14504701309 [Consulta 15/01/2019].

Domínguez-Aroca, M. I. (2017). La biblioteca y las competencias informacionales en el currículo de los estudiantes de ciencias, medicina y ciencias de la salud de la universidad de Alcalá. El profesional de la información, 26(3). https://doi.org/10.3145/epi.2017.may.18

Dorner, J. L.; Taylor, S. E.; Hodson-Carlton, K. (2001). Faculty-librarian collaboration for nursing information literacy: a tiered approach. Reference Services Review, 29(2), 132-141. https://doi. org/10.1108/00907320110394173

Fernández Valdés, M. M.; Zayas, R (2016). Programa de Alfabetización Informacional del Sistema Nacional de Salud cubano: aciertos y desaciertos en su aplicación. Ciencias de la Información, 47(3), 43-48. 
Ferrari, A. (2013). DIGCOMP: A framework for developing and understanding digital competence in Europe. Recuperado de: http://digcomp.org.pl/wp-content/ uploads/2016/07/DIGCOMP-1.0-2013.pdf [Consulta: 15/01/2019].

García Martínez, M. (2010). Competencia informacional de los estudiantes de enfermería del campus Terres de I'Ebre para buscar y seleccionar información académica en internet [Tesis doctoral]. Tarragona: Universitat Rovira i Virgili.

García Martínez, M.; Lleixà Fortuño M.; Nieto de la Fuente C.; Albacar Ribóo N. (2011) Competencia informacional en enfermería y otros profesionales de la salud. Index Enfermería. 20(4), 257-261. Recuperado de: http://scielo.isciii.es/scielo.php?script=sci arttext\&pid $=$ S1132-12962011000300010\&lang $=$ pt [Consulta: 15/01/2019].

Gomes Almeida, M. D. G.; Hernández Pérez, A. (2013). La integración curricular de la Alfabetización Informacional (ALFIN) en las universidades españolas: experiencias de tres modelos distintos. VI Encontro Ibérico edicic 2013: globalização, ciência, informação atas. Recuperado de: https://e-archivo.uc3m.es/bitstream/handle/10016/17965/Gra\%C3\%A7a_tony_edicic_publicado.pdf?sequence $=1 \&$ isAllowed $=y$ [Consulta 15/01/2019].

Gómez-Hernández, J. A.; Benito Morales, F. (2001). De la formación de usuarios a la alfabetización informacional. SCIRE, 7(2), 53-83. Recuperado de: http:// eprints.rclis.org/20758/1/alfin\%20scire\%202001.pdf [Consulta 15/01/2019].

González Fernández-Villavicencio, N. (2010). La Biblioteca de la Universidad de Sevilla, de la FU (Formación de usuarios) a las CI2 (Competencias informáticas e informacionales). Veintitantas experiencias ALFIN y una canción esperanzada. ALFARED. Recuperado de http://www.alfared.org/sites/www.alfared.org/ files/u49/08-nievesgonz\%C3\%A1lez.pdf [Consulta 15/01/2019]

González Fernández-Villavicencio, N. (2016). La competencia digital en el aula. Recuperado de: https://www. slideshare.net/nievesglez/competencias-digitales-enel-aula?from_action=save [Consulta 15/01/2019].

González-Valiente, C. L.; Sánchez-Rodríguez, Y.; Lezcano-Pérez, Y. (2013). Propuesta de un programa de alfabetización informacional para los estudiantes de la Universidad de la Habana. Bibliotecas. Anales de Investigación, (8-9), 121-131.

Guerra Martín, M. D.; Lima Serrano, M.; Zambrano Domínguez, E. M.; Lima Rodríguez, J. S. (2014). ¿ Es efectivo el aprendizaje sobre alfabetización en información para estudiantes de enfermería?. Enfermería Global, 13(4), 90-102. https://doi.org/10.6018/eglobal.13.4.182541

Humboldt State University Library (1999). Nursing information literacy Skills, as adapted from a document by Corryn Crosby-Muilenburg. Humboldt, CA: Humboldt State University Library.
Jacobs, S. K.; Rosenfeld, P.; Haber, J. (2003). Information literacy as the foundation for evidence-based practice in graduate nursing education: a curriculum-integrated approach. Journal of Professional Nursing, 19(5), 320328. https://doi.org/10.1016/S8755-7223(03)00097-8

Jacobsen, H. E.; Andenæs, R. (2011). Third year nursing students' understanding of how to find and evaluate information from bibliographic databases and Internet sites. Nurse education today, 31(8), 898-903. https:// doi.org/10.1016/j.nedt.2011.01.003

Janke, R.; Pesut, B.; Erbacker, L. (2012). Promoting information literacy through collaborative service learning in an undergraduate research course.Nurse Education Today, 32(8), 920-923. https://doi.org/10.1016/j. nedt.2011.09.016

Lau, J.; Cortés, J. (2006). Directrices para el desarrollo de habilidades informacionales: propuesta IFLA abreviada. Revista Brasileira de Biblioteconomia e Documentação, 2(1), 83-104.

Limberg, L.; Alexandersson, M.; Lantz-Andersson, A. (2008). What matters? shaping meaningful learning through teaching information. Libri, 58, 82-91. Recuperado de: http://www.researchgate.net/publication/228912149 What matters_Shaping_meaningful_learning_through_teaching_information_literacy [Consulta 15/01/2019].

Manso Perea, C.; Cuevas Cerveró, A; Martínez Miguel, E.; García-Carpintero Blas, E. (2015). Competencias informacionales en ciencias de la salud una propuesta formativa para estudiantes de Grado en enfermería. Revista Ibero-americana de Ciência da Informação (RICI), 8(1), 1-13. Recuperado de: http://hdl.handle. net/10760/32547 [Consulta 15/01/2019].

Ortoll Espinet, E. (2004). La competencia informacional en las ciencias de la salud: una visión desde las universidades españolas. Revista Española de Documentación Científica. 27(2), 221-241.

Özbıçakçı, Ş., Gezer, N., \& Bilik, Ö. (2015). Comparison of effects of training programs for final year nursing students in Turkey: Differences in self-efficacy with regard to information literacy. Nurse education today, 35(2). https://doi.org/10.1016/j.nedt.2014.10.008

Pinto-Molina, M.; Puertas-Valdeiglesias, S. (2012). Autoevaluación de la competencia informacional en los estudios de psicología desde la percepción del estudiante. Anales de Documentación, 15(2), 1-15. Recuperado de: http://revistas.um.es/analesdoc/article/ view/151661 [Consulta 15/01/2019].

Pirela Morillo, J.; Vera, C.; de Jesús, J. (2014). El desarrollo de competencias informacionales en estudiantes universitarios: Experiencia y perspectivas en dos universidades latinoamericanas. Investigación bibliotecológica, 28(64), 145-172. https://doi.org/10.1016/ S0187-358X(14)70913-X

Rockman, I. F. and Associates (2004). Integrating information literacy into the higher education curriculum: practical models for transformation. San Francisco: Jossey-Bass. 
Royal College of Nursing (RCN). (2011). Finding, using, and managing information: nursing, midwifery, health and social care information literacy competences. Londres: RCN. Recuperado de: https://www.rcn.org.uk/ professional-development/publications/pub-003847 [Consulta 15/01/2019].

Salvador Oliván, J.; Orera Orera, L.; Cantín Luna, M.; Escar Hernández, E.; Lamarca Langa, G.; Vidal Bordés, F. (2011). La presencia de competencias en información en los títulos de grado de la Universidad de Zaragoza. Anales de Documentación. 14(2), 1-16 Recuperado de: http://revistas.um.es/analesdoc/article/view/114981 [Consulta 15/01/2019].

Sánchez Díaz, M. (2010). Competencias informacionales en la formación de las BioCiencias en Cuba. Granada: Universidad de Granada.

Stombaugh, A.; Sperstad, R.; VanWormer, A., Jennings, E., Kishel, H., \& Vogh, B. (2013). Using lesson study to integrate information literacy throughout the curriculum. Nurse educator, 38(4), 173-177. https://doi. org/10.1097/NNE.0b013e318296db56
Tarrant, M.; Dodgson, J. E.; Law, B. V. (2008). A curricular approach to improve the information literacy and academic writing skills of part-time post-registration nursing students in Hong Kong. Nurse Education Today, 28(4), 458-468. https://doi.org/10.1016/j. nedt.2007.08.001

Verhey, M. P. (1999). Information literacy in an undergraduate nursing curriculum: development, implementation, and evaluation. Journal of Nursing Education, 38(6), 252-259.

Wallace, M. C., Shorten, A., Crookes, P. A., McGurk, C.; Brewer, C. (1999). Integrating information literacies into an undergraduate nursing programme. Nurse Education Today, 19(2), 136-141. https://doi. org/10.1054/nedt.1999.0621

Wang, L. (2011) An information literacy integration model and its application in higher education. Reference Services Review, 39(4), 703-720. https://doi. org/10.1108/00907321111186703 